\title{
Independent measurement of the top quark mass and the light- and bottom-jet energy scales at hadron colliders
}

\author{
F. Fiedler ${ }^{\mathrm{a}, \mathrm{b}}$ \\ Ludwig-Maximilians-Universität München, Department für Physik, Am Coulombwall 1, 85748 Garching, Germany
}

Received: 21 June 2007 / Revised version: 11 October 2007 /

Published online: 16 November 2007 - (C) Springer-Verlag / Società Italiana di Fisica 2007

\begin{abstract}
A method for the simultaneous determination of the energy scales for bottom-quark jets and light jets, the jet energy resolution, and the top quark mass at hadron colliders is presented. The method exploits the unique kinematics of events with top-antitop pair production, where one of the top quarks involves a leptonic and one a hadronic $W$ boson decay. The paper shows a feasibility study of how this simultaneous measurement can be performed at the upcoming LHC experiments ATLAS and CMS.
\end{abstract}

\section{Introduction}

Precise knowledge of the energy scale for $b$-quark jet reconstruction will be crucial for many measurements at the ATLAS and CMS experiments at the upcoming LHC collider at CERN, including decays of the top quark, Higgs boson, and supersymmetric particles. The determination of the absolute energy scale for calorimeter jets has however proved to be a challenging task for the CDF and D0 experiments at the Fermilab Tevatron collider. For light jets, this scale can be determined from collider data using events containing an isolated energetic photon balanced by a jet $[1,2]$. In contrast to the calibration of charged-lepton reconstruction, massive resonances decaying to a jet-jet final state cannot readily be used for jet energy calibration; for example, the reconstruction of $Z \rightarrow b \bar{b}$ decays is difficult because of the large backgrounds. The only exception is the reconstruction of hadronic $W$ decays in events containing a top-antitop pair, where one of the top quark decays (charge conjugate modes are implicitly included throughout this paper) involves a hadronic, and the other a leptonic $W$ decay (" $\ell+$ jets $t \bar{t}$ events"). Based on such events, both CDF and D0 have performed simultaneous measurements of the top quark mass and the calorimeter jet energy scale $S_{j}$ for light-quark jets [3-7]. No separate determination of the $b$-quark jet energy scale is available so far, and in these measurements, the uncertainty on the relative difference between the energy scales for light-quark and $b$-quark jets leads to one of the largest remaining systematic uncertainties on the top quark mass.

In this paper, a novel measurement technique is presented that allows a simultaneous determination of the top

\footnotetext{
a Current address: Institut für Physik, Universität Mainz, Staudingerweg 7, 55099 Mainz, Germany

b e-mail: ffiedler@uni-mainz.de
}

quark mass $m_{t}$, light-quark jet energy scale $S_{j}$, relative light- to $b$-quark jet energy scale $S_{b}$, and the jet energy resolution $R$ from $\ell+$ jets $t \bar{t}$ events recorded at a hadron collider. The energy scale $S_{j}$ is defined like for example in [8], i.e. a scale larger than 1.0 corresponds to a calorimeter response larger than unity, which means that the reconstructed jet energy is too large on average. The factor $S_{j}$ applies to all jets, and an additional factor $S_{b}$ is introduced to describe differences between light-quark and $b$-quark jets. This definition implies that in any given measured event, light-quark jet energies have to be divided by a factor $S_{j}$ (and $b$-quark jet energies by a factor $S_{j} \times S_{b}$ ) to reconstruct the top quark mass. Thus, for example, the mass reconstructed for the top quark with the hadronic $W$ decay decreases when the $S_{b}$ hypothesis is increased. The method to determine all four parameters relies on the fact that the dependence on $S_{b}$ is in general different for the two reconstructed invariant top quark masses per event. This is because the transverse momentum (relative to the beam) of the neutrino from the leptonic $W$ decay is reconstructed by imposing transverse momentum balance of the event, which means that it depends on $S_{b}$ if the magnitude of the vector sum of the two $b$-quark transverse momenta is large, while there is no such dependence for the hadronic $W$ decay. Events with small vector sum of the two $b$-quark transverse momenta cannot contribute independent information on $m_{t}$ and $S_{b}$.

In the following it is assumed that calorimeter noise subtraction and the full calorimeter calibration up to a constant scale (which may be different for light and $b$-quark jets) have been performed before the technique described here is applied. Noise studies can be performed with events recorded with minimal trigger requirements; the pre-calibration can be based on the transverse momentum balance in dijet events. In broad terms, the method to determine the top quark mass and absolute jet energy scales then comprises 
the following steps: A sample of candidate $\ell+$ jets $t \bar{t}$ events that contain independent information on $m_{t}$ and $S_{b}$ is selected. To measure the four parameters $m_{t}, S_{j}, S_{b}$, and $R$, estimators are calculated for each selected event. The number of estimators does not have to be equal to the number of parameters to be determined; in the analysis described here, the four parameters are measured using three estimators $m_{t}^{\text {reco }}, S_{j}^{\text {reco }}$, and $S_{b}^{\text {reco }}$ which are chosen so that they are related with $m_{t}, S_{j}$, and $S_{b}$. Functions are derived to describe the expected estimator distributions (templates) for any given set of assumed values of the parameters $m_{t}, S_{j}, S_{b}$, and $R$. The measured estimator distributions in the data are then compared to these fitted templates, and the $m_{t}, S_{j}, S_{b}$, and $R$ values and their uncertainties and correlations are determined in a fit. To validate the procedure, the analysis is performed on simulated pseudo-experiments. To be precise, since the method is based on a fit to simulated templates, it determines the ratio of energy scales in data and simulated events.

The method should be applicable to both the LHC and the Tevatron experiments; as a concrete example, the experimental situation at the LHC (proton-proton collisions at a center-of-mass energy of $14 \mathrm{TeV}$ ) is considered in the following. Signal $t \bar{t} \ell+$ jets events are generated at parton level and smeared according to a parameterized detector resolution. No gluon radiation and no backgrounds are included, and it is assumed that the two $b$-quark jets are identified unambiguously, i.e., the efficiency $\epsilon_{b}$ for $b$-quark jet identification may be smaller than unity, but no light jets are misidentified. The aim of this paper is thus to establish the feasibility of the calibration technique for such an idealized case; conditions that are closer to reality and systematic uncertainties will be addressed in a later publication. In general, it cannot be assumed that the $b$-quark jet energy scale measurement is unbiased by the restriction to those $b$-quark jets that are identified. However, this is not a drawback as most applications, i.e. measurements for which a $b$-quark jet energy scale determination is needed, will also use identified $b$ jets. Residual biases due to differences in event topologies between top-antitop events and other event classes will have to be studied with simulated events, but can be considered a second-order effect.

The paper is organized as follows: Sect. 2 contains a general discussion of the kinematic reconstruction of $\ell+$ jets $t \bar{t}$ events used in the measurement technique presented here. The generation of simulated events and the event selection are described in Sect. 3, and results of a toy-Monte Carlo study of the reconstruction method, based on realistic parameters for the resolution of the LHC detectors, are discussed in Sect. 4. Section 5 summarizes the findings and gives an outlook and conclusion.

\section{Kinematic event reconstruction}

This section describes the event-by-event computation of the estimators $m_{t}^{\text {reco }}, S_{j}^{\text {reco }}$, and $S_{b}^{\text {reco }}$. It should be noted that these calculations can be performed for any $\ell+$ jets $t \bar{t}$ candidate event and that the validity of this section is not restricted to the simplifying assumptions made in the generation of events used later in this paper. While the information in templates obtained with more realistic conditions may be somewhat diluted, the general arguments made in this section will still hold.

The signature of a $\ell+$ jets $t \bar{t}$ event in the detector is the presence of four energetic jets (two $b$-quark jets from the top and antitop, and two light-quark jets from the hadronic $W$ decay), an isolated energetic charged lepton (only electrons and muons are considered here, as $\tau$ leptons decay close to the interaction point leading to additional complications), and missing transverse momentum (with respect to a balanced event) due to the undetected neutrino. There may be additional jets from gluon radiation, however such jets often have lower transverse energy than the $t \bar{t}$ decay products. Such jets are not considered here, but a minimum jet transverse energy is always required in the experimental event selection (such a cut is also included in the study described in Sect. 3).

When the masses of the six $t \bar{t}$ decay products are assumed to be known, there are 18 unknowns from the 6 final-state particle 3-momenta per event, and in addition 3 unknowns corresponding to the estimators $m_{t}^{\text {reco }}$, $S_{j}^{\text {reco }}$, and $S_{b}^{\text {reco }}$ to be calculated on an event-by-event basis. There are 17 measurements in each event corresponding to the five 3 -momenta of the four jets and the charged lepton and to the two components of the missing transverse momentum. (In fact, the missing transverse momentum is a derived quantity, calculated from the vector sum of the momenta of all other final-state particles and other "unclustered energy" in the event that is not assigned to finalstate jets. By imposing transverse momentum balance, the neutrino transverse momentum can be identified with the missing transverse momentum, but its reconstructed value is not independent of the reconstructed jet and chargedlepton momenta.) In addition to the above 17 measurements, 4 constraints can be applied in each event, corresponding to the invariant masses of the two $W$ bosons and two top quarks. Given that there are as many measurements as unknowns, the event kinematics can be solved and the three estimators $m_{t}^{\text {reco }}, S_{j}^{\text {reco }}$, and $S_{b}^{\text {reco }}$ calculated.

The correct assignment of jets to final-state quarks is not known a priori, but it is assumed that the two lightquark jets can be assigned unambiguously to the hadronic $W$ decay. The known mass $m_{W}$ of the hadronically decaying $W$ boson yields information on the energy scale $S_{j}$ for light-quark jets. It is assumed that the scale factor is the same for both jets, such that the estimator $S_{j}^{\text {reco }}$ is calculated as

$$
S_{j}^{\mathrm{reco}}=\frac{m_{W}^{\mathrm{raw}}}{m_{W}},
$$

where $m_{W}^{\text {raw }}$ is the mass obtained from the reconstructed jet energies and momenta. For the following discussion, the measured energies and momenta of all jets are divided by the estimator $S_{j}^{\text {reco }}$ obtained in the same event, and the reconstructed missing transverse momentum is adjusted accordingly.

There are two possibilities for combining the two lightquark jets with one of the $b$-quark jets to reconstruct a top- 
quark decay. To proceed, one can either select one combination (as done in Sect. 3), or consider both of them, possibly together with a suitable relative weight. For a given combination, a scan over $S_{b}^{\text {reco }}$ values is performed. Given an assumed value of $S_{b}^{\text {reco }}$, the reconstructed $b$-quark jet energies and momenta are scaled accordingly, and the missing transverse momentum is adjusted and taken as transverse momentum of the neutrino from the leptonic $W$ decay. The longitudinal neutrino momentum $p_{\nu}^{z}$ is then obtained from the known mass $m_{W}$ of the leptonically decaying $W$ as

$$
\begin{aligned}
& m_{W}^{2}=2 E_{\ell} \sqrt{\left(p_{\nu}^{\mathrm{T}}\right)^{2}+\left(p_{\nu}^{z}\right)^{2}}-2\left(p_{\ell}^{\mathrm{T}} \cdot p_{\nu}^{\mathrm{T}}+p_{\ell}^{z} p_{\nu}^{z}\right) \\
& \Leftrightarrow p_{\nu}^{z}=p_{\ell}^{z} \frac{\frac{1}{2} m_{W}^{2}+p_{\ell}^{\mathrm{T}} \cdot p_{\nu}^{\mathrm{T}}}{E_{\ell}{ }^{2}-p_{\ell}^{z 2}} \\
& \pm \sqrt{\left(p_{\ell}^{z} \frac{\frac{1}{2} m_{W}^{2}+p_{\ell}^{\mathrm{T}} \cdot p_{\nu}^{\mathrm{T}}}{E_{\ell}{ }^{2}-p_{\ell}^{z 2}}\right)^{2}+\frac{\left(\frac{1}{2} m_{W}^{2}+p_{\ell}^{\mathrm{T}} \cdot p_{\nu}^{\mathrm{T}}\right)^{2}-E_{\ell}{ }^{2}\left(p_{\nu}^{\mathrm{T}}\right)^{2}}{E_{\ell}{ }^{2}-p_{\ell}^{z 2}}} .
\end{aligned}
$$

If the solutions are real, one obtains one value for the reconstructed mass $m_{t, \text { lep }}^{\text {reco }}$ of the top quark with the leptonic $W$ decay for each $p_{\nu}^{z}$ solution. The reconstructed mass $m_{t \text {, had }}^{\text {reco }}$ of the top quark with the hadronic $W$ decay is also computed for the given $S_{b}$ value. If one finds $m_{t, \text { lep }}^{\text {reco }}=m_{t, \text { had }}^{\text {reco }}$, then this top quark mass and the corresponding $S_{b}^{\text {reco }}$ value can be taken as estimator values for the event. There are up to four solutions per event: two assignments of $b$-quark jets and two solutions to (2).

\section{Simulation and event selection}

The kinematic event reconstruction and the measurement of the four quantities $m_{t}, S_{j}, S_{b}$, and $R$ have been tested with the experimental resolution of the ATLAS and CMS experiments in mind. The ALPGEN [9] program has been used to generate $\ell+$ jets $t \bar{t}$ events in proton-proton collisions at a center-of-mass energy of $14 \mathrm{TeV}$ for true top quark masses between 160 and $190 \mathrm{GeV}$ in steps of $5 \mathrm{GeV}$. To simulate the effect of jet reconstruction in the experiment, the energies of the final-state quarks have been smeared according to a Gaussian resolution whose width $\sigma(E)$ is set to

$$
\sigma(E)=R \sqrt{E},
$$

with values of $R$ between 0.8 and $1.2 \sqrt{\mathrm{GeV}}$ varied in steps of $0.1 \sqrt{\mathrm{GeV}}$ (this corresponds to a resolution $\sigma(E) / E$ between $80 \% / \sqrt{E}$ and $120 \% / \sqrt{E}$ with $E$ in units of $\mathrm{GeV}$ ). The jet energy resolution of the ATLAS and CMS experiments is expected to be of the same order of magnitude [10, 11]. Finally, all jet energies and momenta are multiplied by a factor $S_{j}$ between 0.8 and 1.2 in steps of 0.1 , and independently, another multiplicative scale factor of $S_{b}$ between 0.8 and 1.2 in steps of 0.1 is applied to $b$-quark jet energies and momenta.

A preselection of $\ell+$ jets $t \bar{t}$ candidate events is first applied:
(P1) A minimum charged-lepton transverse energy of $20 \mathrm{GeV}$ is required.

(P2) The pseudorapidity $\eta$ of the charged lepton and all jets must be between -2.5 and 2.5 .

(P3) The angular separation $\Delta \mathcal{R}=\sqrt{(\Delta \eta)^{2}+(\Delta \phi)^{2}}$ between the charged lepton and any jet must be larger than 0.4 , and the same must be true for any jet-jet pair.

(P4) After smearing and energy scaling, the transverse energies of all jets must be larger than $30 \mathrm{GeV}$.

The estimator $S_{j}^{\text {reco }}$ is calculated from the reconstructed mass of the hadronically decaying $W$ as in (1), and all reconstructed jet energies and momenta are scaled by $1 / S_{j}^{\text {reco }}$. Events in which the jet assignment is unambiguous and that contain independent information on the top quark mass and $b$-quark jet energy scale are then selected by these criteria:

(S1) To unambiguously assign the two $b$-quark jets, it is required that exactly one of the $b$-quark jets have an invariant mass together with the light-quark jet pair between 150 and $200 \mathrm{GeV}$. In the following, this $b$-quark jet is assumed to come from the decay of the top quark with the hadronically decaying $W$. For a sample generated with $m_{t}=175 \mathrm{GeV}, S_{j}=S_{b}=1$, and $R=1 \sqrt{\mathrm{GeV}}, 74 \%$ of the events that pass the preselection fulfill this criterion.

(S2) As explained in Sect. 1, events with small magnitude of the vector sum of $b$-quark jet transverse momenta cannot yield independent information on the top quark mass and $b$-quark jet energy scale. Therefore, this magnitude is required to be larger than $50 \mathrm{GeV}$. $80 \%$ of the events that pass cut (S1) are retained.

(S3) Events are only retained if exactly one solution to $(2)$ is found with $0.5<S_{b}^{\text {reco }}<2.0$ and $150 \mathrm{GeV}<$ $m_{t}^{\text {reco }}<200 \mathrm{GeV}$. Thus a choice between several possible estimator values is avoided. Of the events that pass criterion (S2), 38\%, 45\%, and $17 \%$ have 0,1 , and 2 such solutions, respectively. More than 2 solutions per event do not occur. Thus, $45 \%$ of the events that pass selection criterion (S2) also pass this cut.

(S4) Finally, the quantity $\Delta^{\text {reco }}:=\frac{\partial m_{t, \text { lep }}^{\text {reco }}}{\partial S_{b}^{\text {reco }}}-\frac{\partial m_{t, \text { had }}^{\text {reco }}}{\partial S_{b}^{\text {reco }}}$ is obtained during the scan of $S_{b}^{\text {reco }}$ values and taken as a measure of whether an event contains independent information on the top quark mass and $b$-quark jet energy scale. The $\Delta^{\text {reco }}$ distribution in all events that pass cut (S3) is shown in Fig. 1. In events with small $\left|\Delta^{\text {reco }}\right|$ the value of $S_{b}^{\text {reco }}$ has a large uncertainty, and thus events with $\left|\Delta^{\text {reco }}\right|<30 \mathrm{GeV}$ are rejected. The $m_{t}^{\text {reco }}$ and $S_{b}^{\text {reco }}$ templates for events with $\Delta^{\text {reco }}<-30 \mathrm{GeV}$ have a degraded resolution compared with the distributions for $\Delta^{\text {reco }}>$ $+30 \mathrm{GeV}$, and only the latter events are retained. This criterion thus selects events in which the value of $m_{t, \text { lep }}^{\text {reco }}$ decreases much less rapidly than $m_{t \text {, had }}^{\text {reco }}$ or even increases with $S_{b}^{\text {reco }}$. A fraction of $11 \%$ of events passes this final cut, yielding an overall efficiency of $2.9 \%$ after the preselection. 


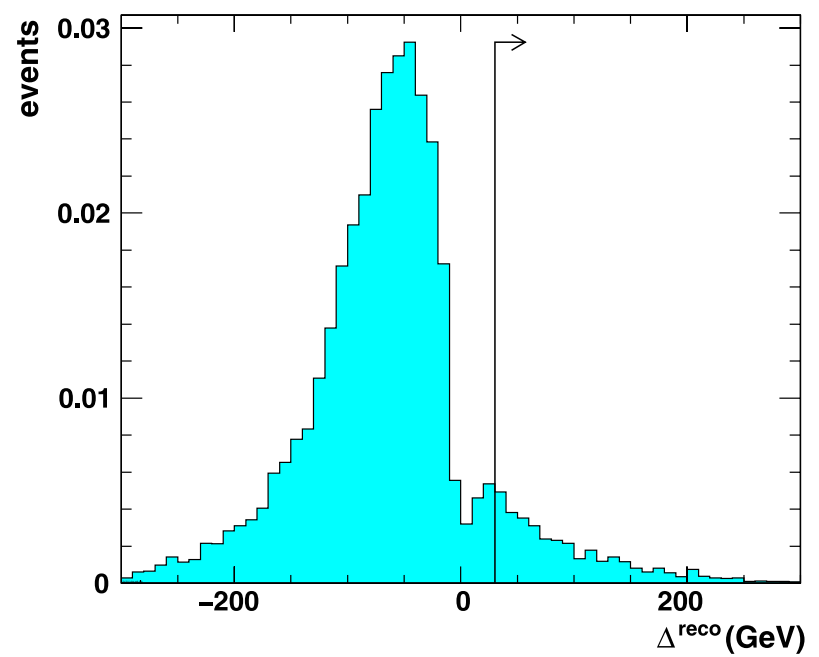

Fig. 1. For simulated events with $m_{t}=175 \mathrm{GeV}, S_{j}=S_{b}=1$, and $R=1 \sqrt{\mathrm{GeV}}$, the distribution of the quantity $\Delta^{\text {reco }}$ for events passing selection cut (S3). The selection applied in (S4) is indicated. The dip at $\Delta^{\text {reco }}=0$ is due to the fact that such events cannot yield a solution for $m_{t, \text { lep }}^{\text {reco }}=m_{t, \text { had }}^{\text {reco }}$

The effect of criteria (S2) and (S4) is illustrated in Fig. 2: Plot (a) shows the $m_{t}^{\text {reco }}$ vs. $S_{b}^{\text {reco }}$ estimator distribution for a sample generated with $m_{t}=175 \mathrm{GeV}$, $S_{j}=S_{b}=1$, and $R=1 \sqrt{\mathrm{GeV}}$ when all selection cuts except (S2) and (S4) are applied; plot (b) shows events that pass the full event selection. It is evident how the $m_{t}^{\text {reco }}$ and $S_{b}^{\text {reco }}$ resolution is superior in those events that pass all selection cuts; the correlation is reduced from -0.76 to -0.62 . Plot (c) shows the $m_{t}^{\text {reco }}$ vs. $S_{b}^{\text {reco }}$ estimator distributions for events passing the full selection for various input values; it can be seen that independent information on $m_{t}$ and $S_{b}$ is contained in the events.

It should be noted that the present analysis is intended as a conceptual study and a proof of principle. Consequently, no optimisation of the cut values in the individual selection criteria has been performed.

Estimator distributions for various choices of input parameters are shown in Fig. 3. This figure shows clearly the sensitivity to a variation of the input parameters.

\section{Template fit}

Estimator distributions have been determined from the simulated events described above for various $m_{t}, S_{j}, S_{b}$, and $R$ input values. To become insensitive to statistical fluctuations of these templates, functions $T_{e}$ have been derived to describe each of the three estimator distributions, $e=m_{t}^{\text {reco }}, S_{j}^{\text {reco }}, S_{b}^{\text {reco }}$. The $m_{t}^{\text {reco }}$ templates are described with a normalized double Gaussian, and normalized single Gaussian functions are used to describe the $S_{j}^{\text {reco }}$ and $S_{b}^{\text {reco }}$ templates. The parameters that describe these functions are themselves taken to be linear functions of the $m_{t}$, $S_{j}, S_{b}$, and $R$ input values. The fact that this simple parameterization cannot describe every detailed aspect of the templates is no limitation for this conceptual study. It will lead to small deviations between fitted and input values, see below; however, the general conclusion that all four quantities can be measured independently of each other remains valid.
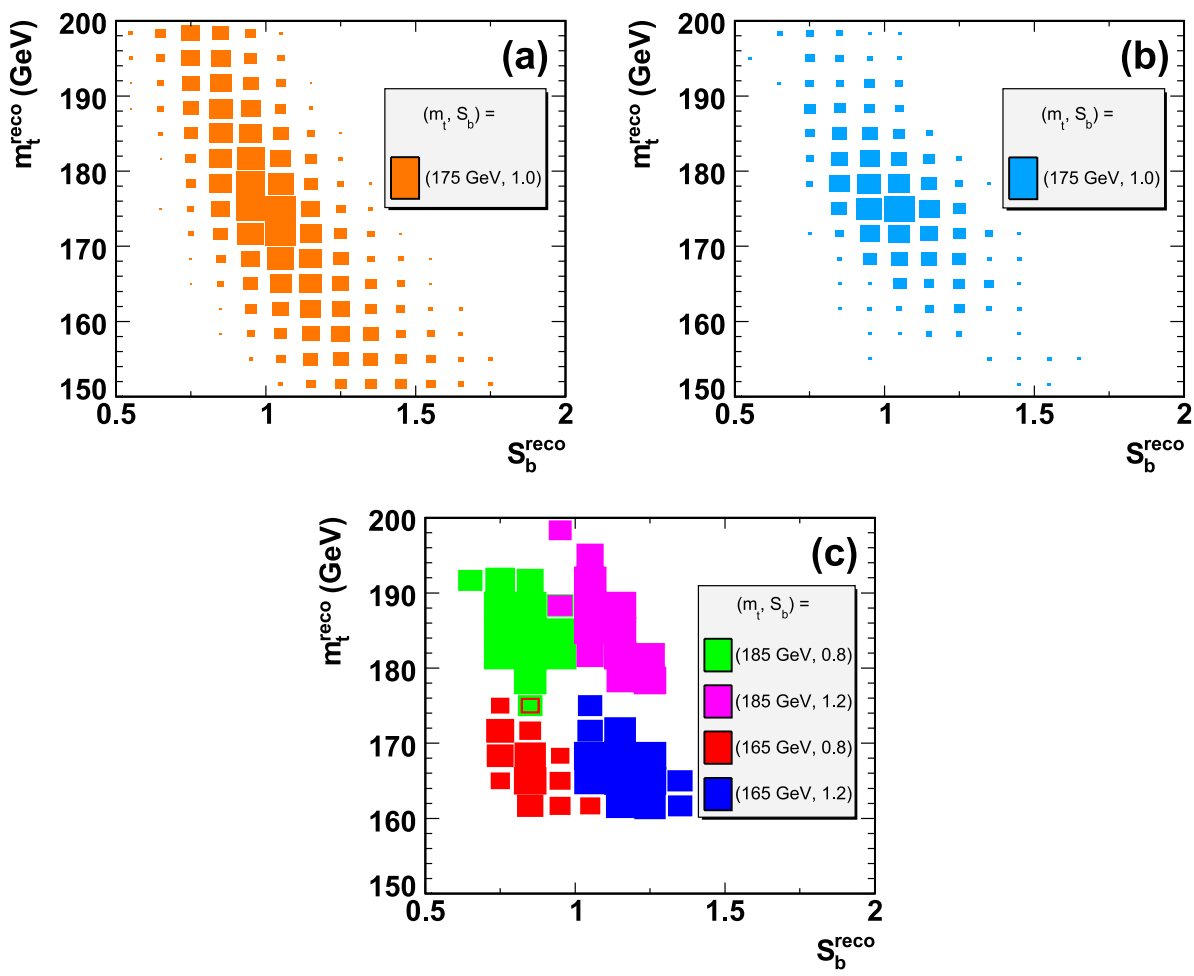

Fig. 2. For simulated events with $m_{t}=$ $175 \mathrm{GeV}, S_{j}=S_{b}=1$, and $R=1 \sqrt{\mathrm{GeV}}$, the two-dimensional $m_{t}^{\text {reco }}$ vs. $S_{b}^{\text {reco }}$ estimator distribution is shown. For plot a, cuts (S2) and (S4) are not applied and for plot $\mathbf{b}$, the full event selection is applied. In c, two-dimensional estimator distributions are shown with separate color codes for $\left(m_{t}, S_{b}\right)$ input values of $(185 \mathrm{GeV}, 0.8)$ : green, $(185 \mathrm{GeV}, 1.2)$ : magenta, $(165 \mathrm{GeV}$, 0.8): red, and (165 GeV, 1.2): blue. The input values of $S_{j}=1$ and $R=1 \sqrt{\mathrm{GeV}}$ have been kept. Here, only those bins are shown that contain at least $2 \%$ of the events. The reconstructed estimator distributions cluster around the input $m_{t}$ and $S_{b}$ values. In all plots, the size of the squares is proportional to the number of entries 

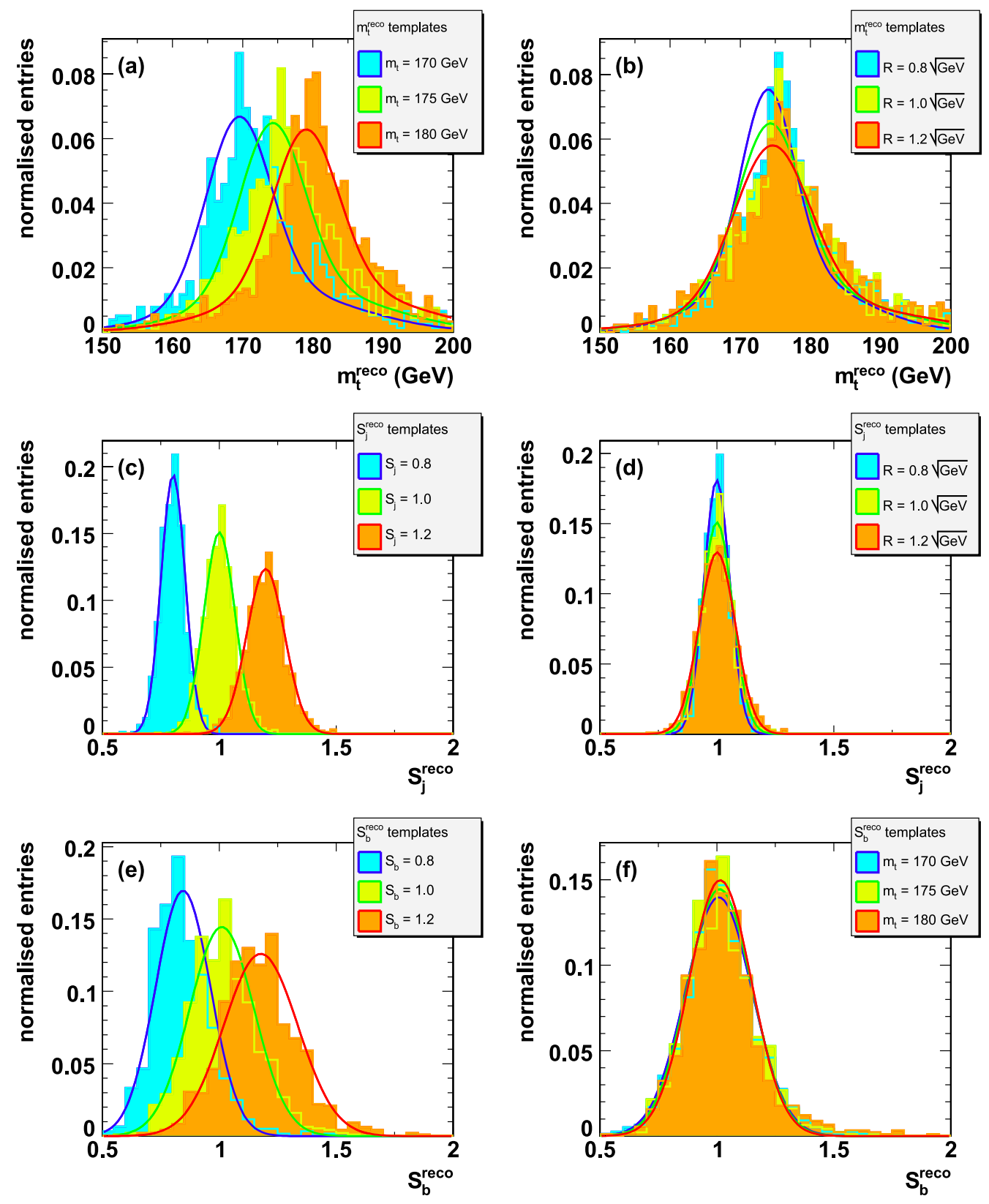

Fig. 3. Estimator distributions for simulated events with different input parameters. All distributions are normalised. Parameter values used in the event generation are $m_{t}=175 \mathrm{GeV}, S_{j}=S_{b}=$ 1 , and $R=1 \sqrt{\mathrm{GeV}}$ except as noted in the plots. Plot a shows how the $m_{t}^{\text {reco }}$ template depends on the input $m_{t}$ value, plot $\mathbf{b}$ shows the dependence on the $R$ value. Similar plots are shown in c and $\mathbf{d}$ for the dependence of the $S_{j}^{\text {reco }}$ distribution on the input $S_{j}$ and $R$ values. In e, the dependence of the $S_{b}^{\text {reco }}$ template on the $S_{b}$ input is visualized, while plot $\mathbf{f}$ shows that there is no large dependence of the $S_{b}^{\text {reco }}$ distribution on the $m_{t}$ input value. The functions used to parameterize the templates as described in Sect. 4 are overlaid

Pseudo-experiments are then performed using simulated events as pseudo-data. A binned likelihood fit is performed to determine $m_{t}, S_{j}, S_{b}$, and $R$ in each pseudoexperiment, with the likelihood $\mathcal{L}$ as a function of $m_{t}, S_{j}$, $S_{b}$, and $R$ hypotheses given by

$$
\begin{aligned}
& -\ln \mathcal{L}\left(m_{t}, S_{j}, S_{b}, R\right) \\
& =-\sum_{\substack{e=m_{t}^{\text {reco }}, S_{j}^{\text {reco }}, S_{b}^{\text {reco }}}} \sum_{i=1}^{n_{\text {bins }}(e)} D_{e}(i) \ln T_{e}\left(m_{t}, S_{j}, S_{b}, R ; i\right) .
\end{aligned}
$$

The first sum is over the three estimator quantities, generically denoted by $e$, and the second sum runs over the bins $i$ of each estimator distribution. The number of pseudodata events in bin $i$ of estimator distribution $e$ is denoted by $D_{e}(i)$, while $T_{e}\left(m_{t}, S_{j}, S_{b}, R ; i\right)$ is the value of the function used to parameterize the template, evaluated at the center of bin $i$ under the assumption of given $m_{t}, S_{j}$, $S_{b}$, and $R$ values. In each pseudo-experiment, the fourdimensional $-\ln \mathcal{L}$ space is scanned, and the values of $m_{t}$, $S_{j}, S_{b}$, and $R$ that minimize $-\ln \mathcal{L}$ are taken as measured values.

For various sets of input parameter values, 1000 pseudoexperiments with 300 events each have been evaluated. For $m_{t}=175 \mathrm{GeV}$, this corresponds to an integrated luminosity of $100 \mathrm{pb}^{-1} / \epsilon_{b}^{2}$ at the LHC, where $\epsilon_{b}$ is the efficiency of identifying a $b$-quark jet. For a given set of inputs, the distributions of measurement values have been fitted with a Gaussian to determine the expected measurement value and its statistical uncertainty, as shown in Fig. 4. With an integrated luminosity of $100 \mathrm{pb}^{-1} / \epsilon_{b}^{2}$, the LHC 

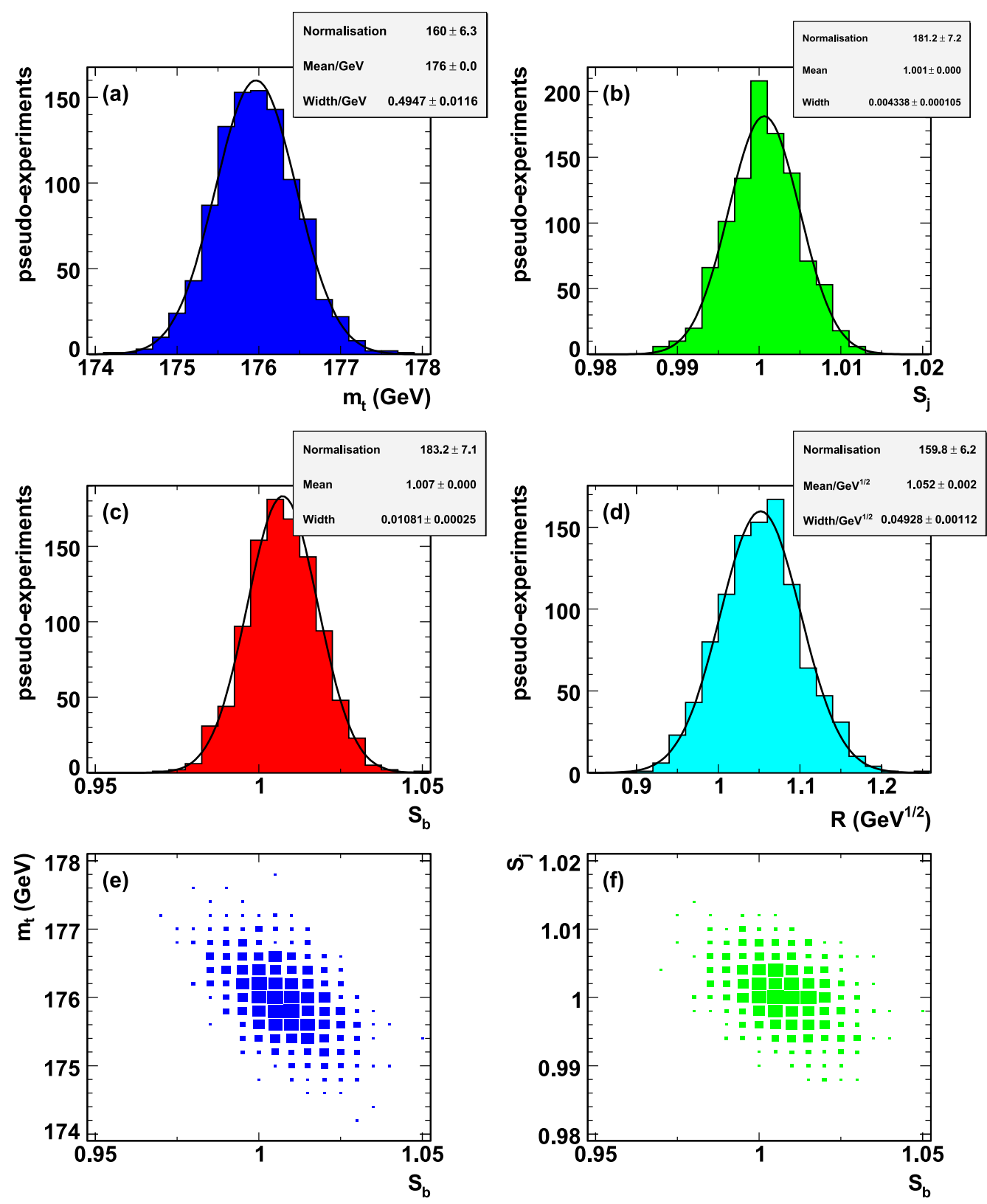

Fig. 4. Distributions of fit results for $m_{t}, S_{j}, S_{b}$, and $R$ (plots $\mathbf{a}, \mathbf{b}, \mathbf{c}$, and $\mathbf{d})$ for pseudo-experiments with 300 events each, generated with parameter values of $m_{t}=$ $175 \mathrm{GeV}, \quad S_{j}=S_{b}=1$, and $R=1 \sqrt{\mathrm{GeV}}$. The correlations between parameters are taken into account. All distributions are fitted with a Gaussian; the resulting mean values and resolutions are given in the plots. The correlation between $m_{t}$ and $S_{b}$ is shown in e, and that between $S_{j}$ and $S_{b}$ in $\mathbf{f}$

experiments will be able to make measurements with the following statistical uncertainties:

$$
\begin{aligned}
\left.\Delta m_{t} \text { (stat. }\right) & =500 \mathrm{MeV} \\
\Delta S_{j}(\text { stat. }) & =0.004 \\
\left.\Delta S_{b} \text { (stat. }\right) & =0.01 \\
\Delta R(\text { stat. }) & =0.05 \sqrt{\mathrm{GeV}} .
\end{aligned}
$$

The information from the pseudo-experiments is then used to obtain the calibration curves in Fig. 5, which show that all four input parameters can be measured independently, i.e. the mean measurement value has a large slope with respect to the input value of the same parameter but no dependence on the values of the other parameters. The correlation matrix between the four measured parameters is given in Table 1.
The bias on the top quark mass and jet energy resolution is not unexpected and is due to the approximative description of the templates by the fitted functions. Such

Table 1. Correlations between the four measured parameters for input values of $m_{t}=175 \mathrm{GeV}, S_{j}=S_{b}=1$, and $R=$ $1 \sqrt{\mathrm{GeV}}$

\begin{tabular}{lcccc}
\hline & $m_{t}$ & $S_{j}$ & $S_{b}$ & $R$ \\
\hline$m_{t}$ & 1.0 & -0.09 & -0.50 & -0.22 \\
$S_{j}$ & -0.09 & 1.0 & -0.38 & -0.11 \\
$S_{b}$ & -0.50 & -0.38 & 1.0 & -0.14 \\
$R$ & -0.22 & -0.11 & -0.14 & 1.0 \\
\hline
\end{tabular}



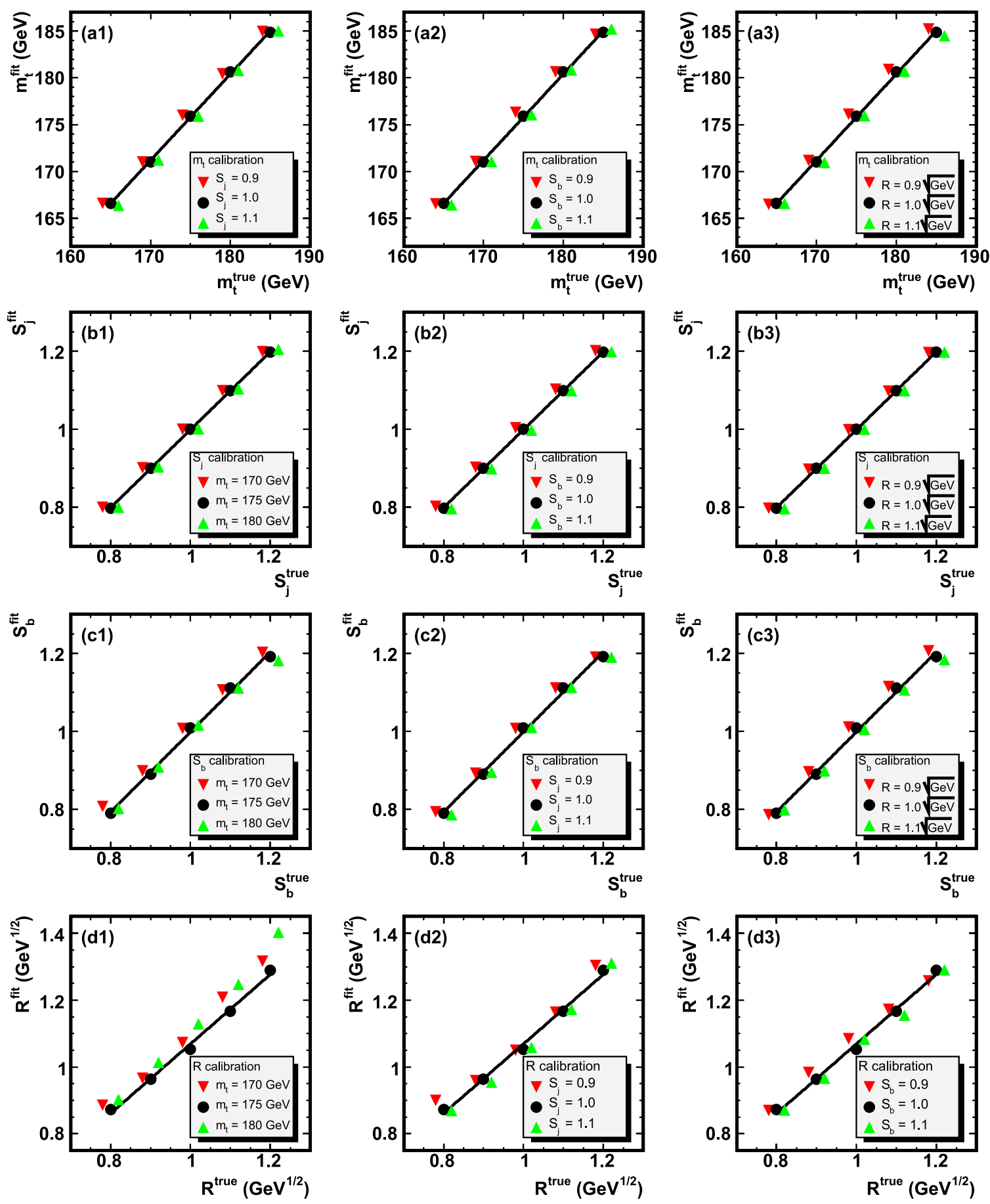

Fig. 5. Calibration curves for the determination of $m_{t}, S_{j}, S_{b}$, and $R$ (plots $\mathbf{a}, \mathbf{b}, \mathbf{c}$, and $\mathbf{d}$ ). For each parameter, calibration curves are shown for various input values for the other three parameters (left, middle, and right columns). Each circle or triangle shows the mean result of pseudo-experiments for a given set of input values. The black circles are identical in all three plots in a row, and the line shows the result of a linear fit to these points. The red downward and green upward triangles show the results of pseudoexperiments when one of the input parameters is varied as specified in each figure. A slight horizontal offset has been applied to the triangles to render them visible. Parameters whose values are not noted explicitly in a plot are set to the default values of $m_{t}=175 \mathrm{GeV}, S_{j}=S_{b}=1$, and $R=1 \sqrt{\mathrm{GeV}}$

effects could be alleviated by using more complicated functions, but that would go beyond the scope of this conceptual study, and the exact template shapes will change anyway when background and full detector simulation are included. In particular, it should be noted that a correction for biases of the raw fit results based on similar calibration curves will always be necessary in a measurement. The bias itself does not necessarily correspond to a systematic 
uncertainty; rather, systematic errors (due e.g. to uncertainties in the simulation of events) can be determined by studying the differences between calibration curves obtained with different simulation parameters.

\section{Conclusion and outlook}

While it is known how the absolute energy scale for lightquark jets can be determined using events taken at hadron collider experiments (using either photon+jet events, balancing the photon and jet in the transverse plane, or $\ell+$ jets $t \bar{t}$ events, exploiting the mass constraint from the hadronically decaying $W$ boson), independent information on the energy scale for $b$-quark jets has so far not been obtained. On the other hand, uncertainties on the relative difference between the energy scales for light- and $b$-quark jets lead to one of the major systematic uncertainties on measurements of the top quark mass at the Tevatron experiments. In this paper, it is shown how $\ell+$ jets $t \bar{t}$ events can be used to determine the top quark mass $m_{t}$, light-quark jet energy scale $S_{j}, b$-quark jet energy scale $S_{b}$, and a jet resolution parameter $R$ independently from each other. The kinematic reconstruction of estimators on an event-by-event basis is discussed, and results of pseudoexperiments with conditions similar to those at the LHC experiments ATLAS and CMS are shown. The technique presented will enable the LHC experiments to measure the top quark mass while at the same time obtaining information on the energy scale for $b$-quark jets. This is crucial for measurements of decays of the Higgs boson or of supersymmetric particles. As increasingly large datasets are accumulated by the Tevatron experiments, the technique may also be used by the CDF and D0 experiments to reduce systematic errors on the top quark mass measurement.

The study presented here is to be viewed as a proof of principle. In the future, the technique will be applied to fully simulated ATLAS events, including background events. Systematic studies need to be performed to assess how much the results depend for example on additional gluon radiation off the initial- or final-state partons. Finally, with the large event samples expected at the LHC experiments in mind, the method has been implemented so far based on a template fit. The determination of the $b$-quark jet energy scale can also be included naturally in the matrix element method used at the Tevatron for top quark mass measurements, which maximizes the statistical sensitivity (but also needs much longer computation time per selected event). Any of these studies would go beyond the scope of this paper but will be addressed in future publications.

Acknowledgements. The author would like to thank S. Menke, R. Nisius, and J. Schwindling for very helpful discussions.

\section{References}

1. A. Bhatti et al., Nucl. Instrum. Methods A 566, 375 (2006) [arXiv:hep-ex/0510047]

2. D0 Collaboration, B. Abbott et al., Nucl. Instrum. Methods A 424, 352 (1999) [arXiv:hep-ex/9805009]

3. CDF Collaboration, A. Abulencia et al., Phys. Rev. D 73, 032003 (2006) [arXiv:hep-ex/0510048]

4. CDF Collaboration, A. Abulencia et al., Phys. Rev. Lett. 96, 022004 (2006) [arXiv:hep-ex/0510049]

5. D0 Collaboration, V.M. Abazov et al., Phys. Rev. D 74, 092005 (2006) [arXiv:hep-ex/0609053]

6. D0 Collaboration, V.M. Abazov et al., Phys. Rev. D 75, 092001 (2007) [arXiv:hep-ex/0702018]

7. CDF Collaboration, A. Abulencia, arXiv:hep-ex/0703045

8. D0 Collaboration, V.M. Abazov et al., Phys. Rev. D 74, 092005 (2006) [arXiv:hep-ex/0609053]

9. M.L. Mangano et al., JHEP 307, 1 (2003)

10. ATLAS Collaboration, ATLAS: Detector and physics performance technical design report. Vol. 1, CERN/LHCC 99-14

11. CMS Collaboration, CMS Physics Technical Design Report, Vol. 1, CERN/LHCC 2006-001 